Supporting information for:

Including and Declaring Structural Fluctuations in the Study of Lanthanide(III) Coordination Chemistry in Solution

Lea Gundorff Nielsen \& Thomas Just Sørensen.*

${ }^{\dagger}$ Nano-Science Center \& Department of Chemistry, University of Copenhagen, Universitetsparken 5, 2100 Copenhagen $\emptyset$, Denmark

\title{
Contents
}

Structure of ligands

Experimental Section 3 


\section{Structure of ligands}<smiles>O=C(O)CN1CCN(CC(=O)O)CCN(CC(=O)O)CCN(CC(=O)O)CC1</smiles>

DOTA

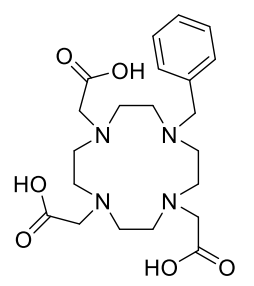

DO3A-benzyl<smiles>NC(=O)CN1CCN(CC(N)=O)CCN(CC(N)=O)CN(CC(N)=O)CC1</smiles>

DOTAM<smiles>CNC(=O)CN1CCN(CC(=O)O)CCN(CC(=O)O)CCN(CC(=O)O)C1</smiles>

DOTA-methylamide<smiles>CNC(=O)CN1CCN(CC(=O)NC)CN(CC(=O)NC)CN(CC(=O)NC)CC1</smiles>

DTMA<smiles>CCCCCNC(=O)CN(CCN1CCN(CC(=O)O)CCN(CC(=O)O)CC1)CC(=O)NCCCCC</smiles>

DOTA-dipentylamide

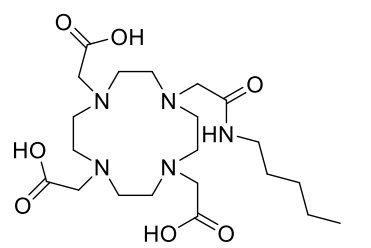

DOTA-pentylamide

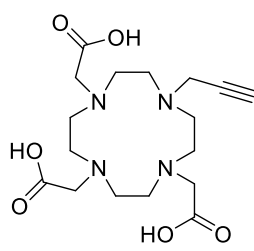

D03A-propargyl

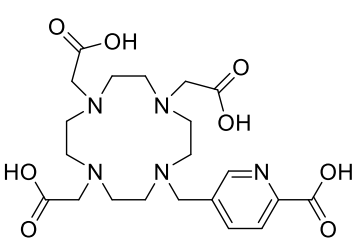

DO3A-PIC

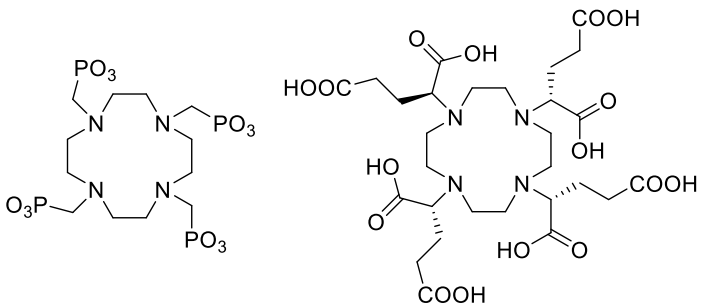

DOTP<smiles>CN(C)C(=O)CN(CCN1CCN(CC(=O)O)CCN(CC(=O)O)CC1=O)CC(=O)O</smiles>

DOTA-2dma

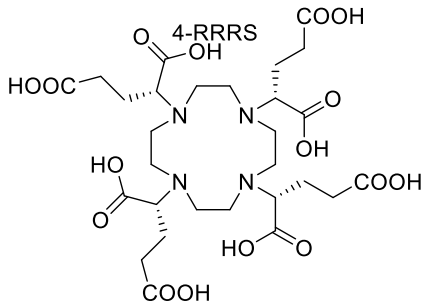

4-RRRR

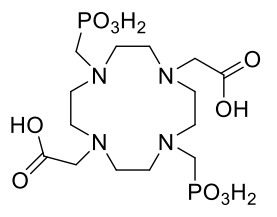

DO2A2P

DOTMAM 


\section{Experimental Section}

\section{General methods for synthesis}

All chemicals and solvents were used as received unless otherwise stated in the specific synthetic procedure. All chemicals purchased from Sigma-Aldrich, Merck and VWR chemicals. Both HPLCgrade and technical grade solvents were used. Water for spectroscopy was deionised and microfiltered using a Milli-Q Millipore water purification system (resistivity $>18.2 \mathrm{M} \Omega \cdot \mathrm{cm}$, total organic carbon (TOC) $<5 \mathrm{ppb}$ ). For thin layer chromatography (TLC) aluminium sheets pre-coated with silica gel 60F (Merck) was used. For column chromatography silica gel with pore size $60 \AA$ and $40-63 \mu \mathrm{m}$ was used. Accurate masses were determined to four decimal places using a Bruker Solarix ESP-MALDIFT-ICR instrument equipped with a $7 \mathrm{~T}$ magnet (the instrument was calibrated using sodium trifluoroacetate cluster ions prior to acquiring the spectrum). ${ }^{1} \mathrm{H}$ NMR and ${ }^{13} \mathrm{C}$ NMR spectra were recorded on a Bruker $500 \mathrm{MHz}$ instrument equipped with a $126 \mathrm{MHz}$ (non-inverse) cryo-probe. ${ }^{1} \mathrm{H}$ NMR spectra of the lanthanide(III) complexes were recorded on a Brucker $500 \mathrm{MHz}$ instrument equipped with a broad-band-probe. All chemical shifts $(\delta)$ are given in parts per million. All NMR were recorded at $25^{\circ} \mathrm{C}$ with deuterated solvents. The residual solvent was used as internal standard.

Optical Spectroscopy: Steady state spectra were recorded on a PTI QuantaMaster8075 from Horiba Scientific using a xenon arc lamp as excitation source. For excitation spectra, emission was detected at $618 \mathrm{~nm}$ and excitation and emission slits were 2 and $6 \mathrm{~nm}$, respectively. For emission spectra, the samples were excited at $393 \mathrm{~nm}$ and excitation and emission slits were set at 8 and $1 \mathrm{~nm}$. Emission intensities in the visible region were corrected for wavelength dependence of the detector sensibility using a factory provided correction file. Lamp intensity fluctuations were corrected by a reference detector. A constant nitrogen flow in the sample chamber was used to avoid fogging of cooled samples. Absorption was kept below 0.1 at excitation wavelengths to avoid in ner filter effects. Starna Scientific $10 \mathrm{~mm}$ quartz cuvettes were used for all samples at ambient temperatures

Luminescence Lifetime Determination: Time-resolved measurements were performed using a PTI Quanta-Master8075 from Horiba Scientific with a xenon flash lamp as the excitation source $(393 \mathrm{~nm})$. Emission was detected at $618 \mathrm{~nm}$. Excitation and emission slits were set at 8 and $8 \mathrm{~nm}$, respectively. Lifetime traces were fitted to a monoexponential decay using the FelixGX (Horiba Scientific) and Origin 2017 (OriginLab) softwares.

\section{1,4,7,10-tetraazacyclododecane-1,4,7,10-tetraacetic acid (DOTA) ${ }^{1}$}

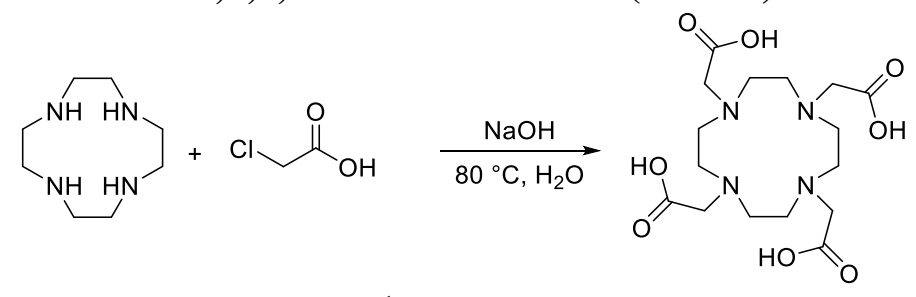

DOTA was prepared as reported by Desreux. ${ }^{1}$

${ }^{1} \mathrm{H}-\mathrm{NMR}\left(500 \mathrm{MHz}, \mathrm{D}_{2} \mathrm{O}\right) \delta 3.64(\mathrm{~s}, 8 \mathrm{H}), 3.31(\mathrm{~s}, 16 \mathrm{H})$.

${ }^{13} \mathrm{C}-\mathrm{NMR}\left(126 \mathrm{MHz}, \mathrm{D}_{2} \mathrm{O}\right) \delta 55.88,49.83$

HRMS (ESI $\left.{ }^{+}\right): \mathrm{m} / \mathrm{z}$ calculated for $\mathrm{C}_{16} \mathrm{H}_{28} \mathrm{~N}_{4} \mathrm{O}_{8}[\mathrm{M}+\mathrm{H}]^{+}:$405.1907; found 405.1990.

\section{1,4,7,10-Tetrakis[(carbamoyl)methyl]-1,4,7,10-tetraazacyclododecane (DOTAM) ${ }^{2}$}




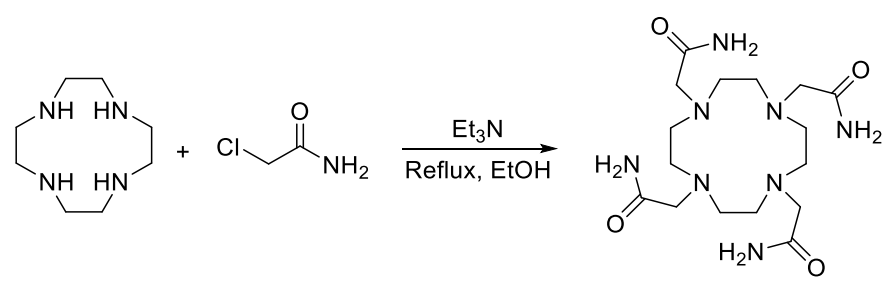

DOTAM was prepared with chloroacetamide instead of bromoacetamide as described in the following procedure. ${ }^{2}$

${ }^{1} \mathrm{H}$ NMR (500 MHz, D $\left.2 \mathrm{O}\right) \delta 3.17$ (s, 8H), 2.72 (s, 16H).

${ }^{13} \mathrm{C}$ NMR (126 MHz, $\left.\mathrm{D}_{2} \mathrm{O}\right) \delta 177.37,57.27,52.46$.

HRMS ES: m/z calculated for $\mathrm{C}_{16} \mathrm{H}_{32} \mathrm{~N}_{8} \mathrm{O}_{4}[\mathrm{M}+\mathrm{H}]^{+}: 401.2619$, found: 401.2614

\section{1,4,7,10-Tetrakis[(N-methylcarbamoyl)methyl]-1,4,7,10-tetraazacyclododecane (DTMA) ${ }^{3}$}
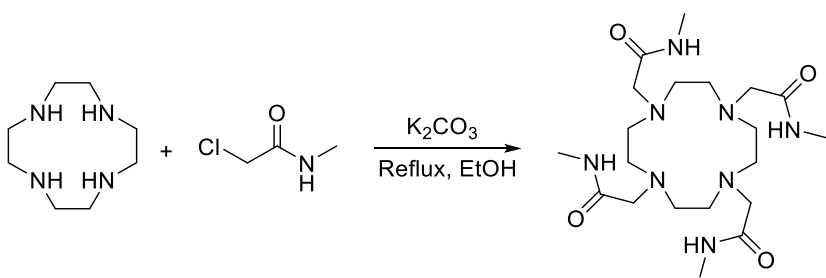

DTMA was synthesized following reported procedures. ${ }^{3}$

${ }^{1} \mathrm{H}$ NMR $\left(500 \mathrm{MHz}, \mathrm{CDCl}_{3}\right) \delta$ 3.06-3.04 (m, 8H) 2.82-2.79 (m, 12H), 2.64-2.63 (m, 16H).

${ }^{13} \mathrm{C}$ NMR $\left(126 \mathrm{MHz}, \mathrm{CDCl}_{3}\right) \delta 59.31,53.45,26.37$.

HRMS ES: $\mathrm{m} / \mathrm{z}$ calculated for $\mathrm{C}_{20} \mathrm{H}_{40} \mathrm{~N}_{8} \mathrm{O}_{4}[\mathrm{M}+\mathrm{H}]^{+}:$457.3245, found: 457.3267

\section{1,7-Bis(benzyloxycarbonyl)-1,4,7,10-tetraazacyclododecane ${ }^{4}$}
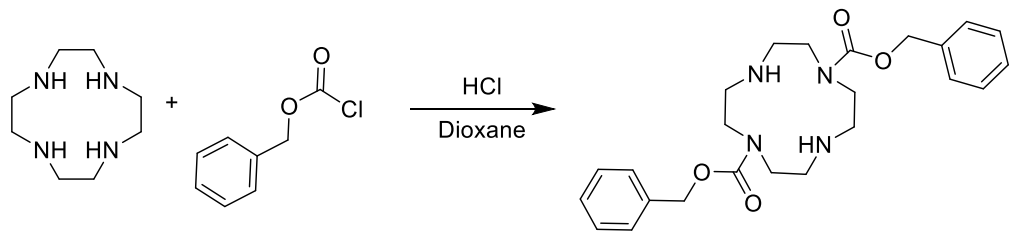

The protected cyclen was prepared following reported literature procedures. ${ }^{4}$

${ }^{1} \mathrm{H}-\mathrm{NMR}\left(500 \mathrm{MHz}, \mathrm{CDCl}_{3}\right) \delta 7.37(\mathrm{~m}, 10 \mathrm{H}), 5.17(\mathrm{~s}, 4 \mathrm{H}), 5.05(\mathrm{~s}, 2 \mathrm{H}), 3.48(\mathrm{~m}, 8 \mathrm{H}), 2.98-2.79(\mathrm{q}$, $8 \mathrm{H})$.

${ }^{13} \mathrm{C}-\mathrm{NMR}\left(126 \mathrm{MHz}, \mathrm{CDCl}_{3}\right) \delta 156.48,136.96,129-128,67.3,67.1,51.31,50.97,50.92,50.41$, $50.30,49.72,49.10,48.78$

HRMS (ESI $\left.{ }^{+}\right): \mathrm{m} / \mathrm{z}$ calculated for $\mathrm{C}_{24} \mathrm{H}_{32} \mathrm{~N}_{4} \mathrm{O}_{4}[\mathrm{M}+\mathrm{H}]^{+}: 441.2496$; found: 441.2519 . 
1,7-Bis(benzyloxycarbonyl)-4,10-bis(hexylcarbamoylmethyl)-1,4,7,10-tetraazacyclododecane 5

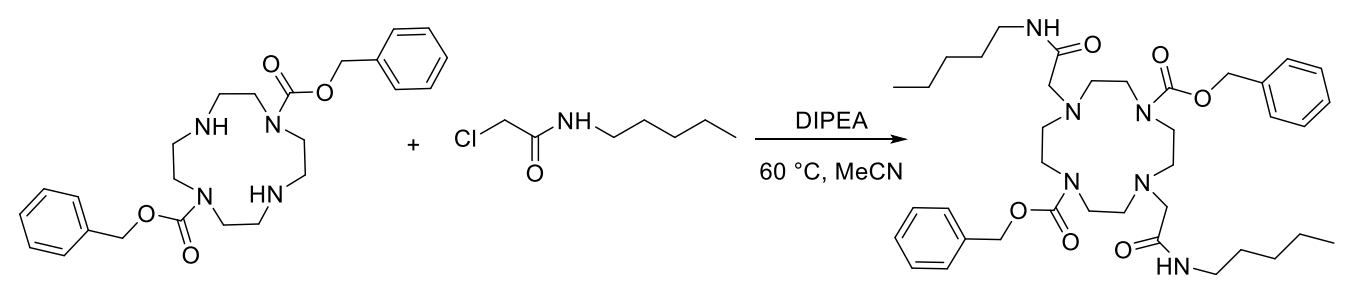

The compound was synthesized following literature procedure for similar compounds but with 2chloro-N-pentylacetamide as the reagent. ${ }^{5}$

${ }^{1} \mathrm{H}$ NMR $\left(500 \mathrm{MHz}, \mathrm{CD}_{3} \mathrm{CN}\right) \delta 7.34(\mathrm{~s}, 10 \mathrm{H}), 5.07$ (s, 4H), $3.44(\mathrm{~s}, 8 \mathrm{H}), 3.06(\mathrm{~s}, 8 \mathrm{H}), 2.72(\mathrm{~s}, 8 \mathrm{H})$, $1.42(\mathrm{~s}, 4 \mathrm{H}), 1.32-1.23(\mathrm{~m}, 9 \mathrm{H}), 0.89-0.83(\mathrm{~m}, 6 \mathrm{H})$.

${ }^{13} \mathrm{C}$ NMR $\left(126 \mathrm{MHz}, \mathrm{CD}_{3} \mathrm{CN}\right) \delta 156.07,136.70,127.97,127.41,127.19,66.20,38.10,28.67$, $28.41,21.60,12.86$.

HRMS (ESI $\left.{ }^{+}\right): \mathrm{m} / \mathrm{z}$ calculated for $\mathrm{C}_{38} \mathrm{H}_{58} \mathrm{~N}_{6} \mathrm{O}_{6}[\mathrm{M}+\mathrm{H}]^{+}:$: 695.4490; found: 695.4494.

\section{1,7-bis(hexylcarbamoylmethyl)-1,4,7,10-tetraazacyclododecane ${ }^{5}$}

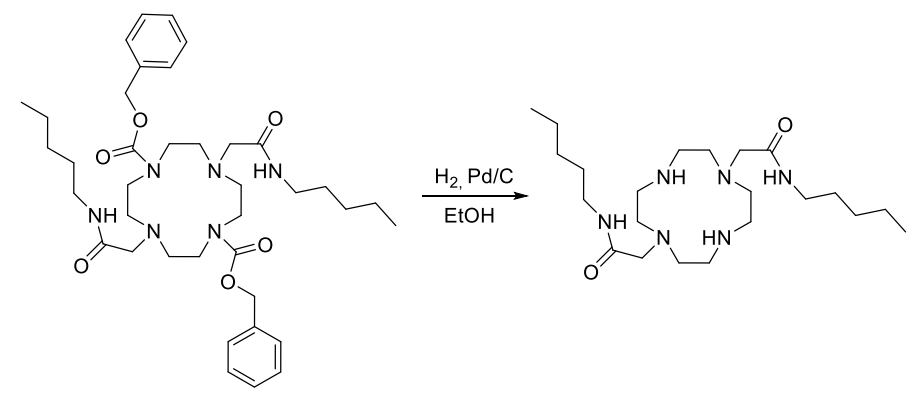

The compound was synthesized following literature procedure for deprotection of a Cbz-group with hydrogenation and $\mathrm{Pd} / \mathrm{C} .^{5}$

${ }^{1} \mathrm{H}$ NMR (500 MHz, $\left.\mathrm{CDCl}_{3}\right) \delta 3.42(\mathrm{~m}, 8 \mathrm{H}), 2.77(\mathrm{~m}, 16 \mathrm{H}), 1.51(\mathrm{~m}, 4 \mathrm{H}), 1.30(\mathrm{~m}, 8 \mathrm{H}), 0.89$ (m, $6 \mathrm{H})$.

${ }^{13} \mathrm{C}$ NMR $\left(126 \mathrm{MHz}, \mathrm{CDCl}_{3}\right) \delta$ 60.23, 53.24, 46.60, 39.56, 29.40, 29.24, 22.42.

HRMS ES: m/z calculated for $\mathrm{C}_{22} \mathrm{H}_{46} \mathrm{~N}_{6} \mathrm{O}_{2}[\mathrm{M}+\mathrm{H}]^{+}: 427.3755$, found: 427.3764 
1,7-Bis(carboxymethyl)-4,10-bis(hexylcarbamoylmethyl)-1,4,7,10-tetraazacyclododecane ${ }^{5,6}$
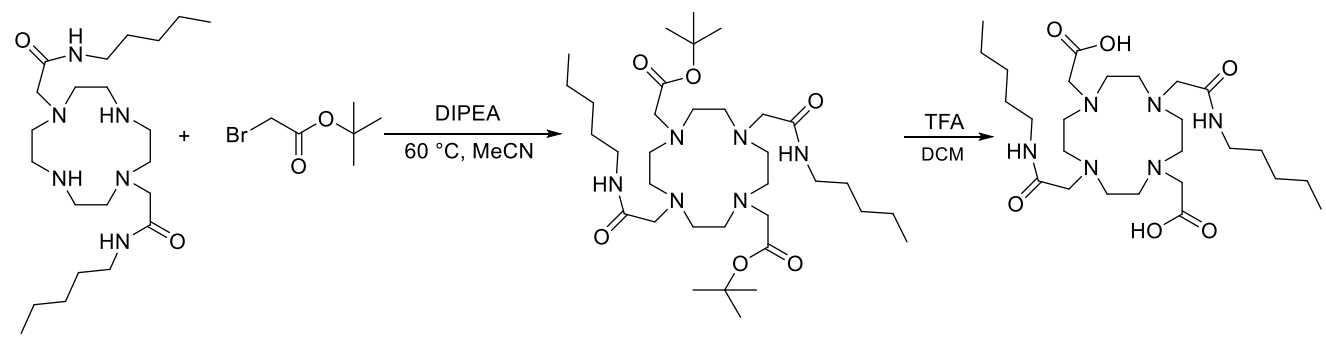

Tert-butyl bromoacetate was attached to the cyclen ring as reported in literature. ${ }^{5}$ The tert-butyl group was removed by TFA as previously reported from our group. ${ }^{6}$

${ }^{1} \mathrm{H}$ NMR $\left(500 \mathrm{MHz}, \mathrm{D}_{2} \mathrm{O}\right) \delta 3.86-3.80(\mathrm{~m}, 4 \mathrm{H}), 3.50(\mathrm{~m}, 12 \mathrm{H}), 3.20(\mathrm{~m}, 8 \mathrm{H}), 3.02(\mathrm{~m}, 4 \mathrm{H}), 1.53$ (p, 4H), $1.36-1.28(\mathrm{~m}, 7 \mathrm{H}), 0.89(\mathrm{t}, 5 \mathrm{H})$.

${ }^{13} \mathrm{C}$ NMR $\left(126 \mathrm{MHz}, \mathrm{D}_{2} \mathrm{O}\right) \delta 55.74,51.20,48.27,39.50,28.47,28.17,21.71,13.29$.

HRMS ES: $\mathrm{m} / \mathrm{z}$ calculated for $\mathrm{C}_{26} \mathrm{H}_{50} \mathrm{~N}_{6} \mathrm{O}_{6}[\mathrm{M}+\mathrm{H}]^{+}:$543.3864, found: 543.3867 , $[\mathrm{M}+\mathrm{Na}]^{+}: 565.3684$, found: 365.3691

\section{1,4,7-tris(carboxymethyl)- 10-Benzyl-1,4,7,10-tetraazacyclododecane ${ }^{7}$}

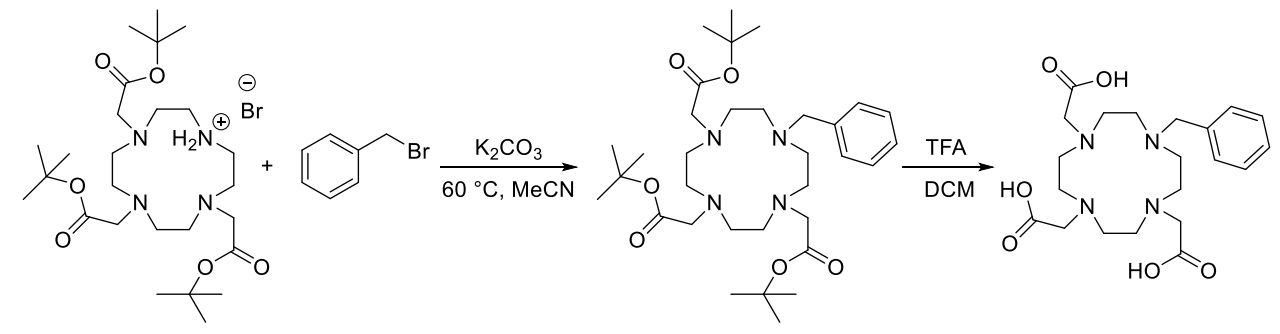

The compound was prepared following reported procedures but with $\mathrm{K}_{2} \mathrm{CO}_{3}$ instead of $\mathrm{Na}_{2} \mathrm{CO}_{3}$. $^{7}$

${ }^{1} \mathrm{H}$ NMR (500 MHz, $\left.\mathrm{CDCl}_{3}\right) \delta$ 7.68-7.40 (m, 5H) 4.20-3.01 (m, 24H).

${ }^{13} \mathrm{C}$ NMR $\left(126 \mathrm{MHz}, \mathrm{D}_{2} \mathrm{O}\right) \delta 132.84,130.24,129.37,56.54,54.16,54.86,50.09$

HRMS ES: $\mathrm{m} / \mathrm{z}$ calculated for $\mathrm{C}_{21} \mathrm{H}_{32} \mathrm{~N}_{4} \mathrm{O}_{6}[\mathrm{M}+\mathrm{H}]^{+}$: 437.2394, found: 437.2427, $[\mathrm{M}+\mathrm{Na}]^{+}: 459.2214$, found: 459.2247 


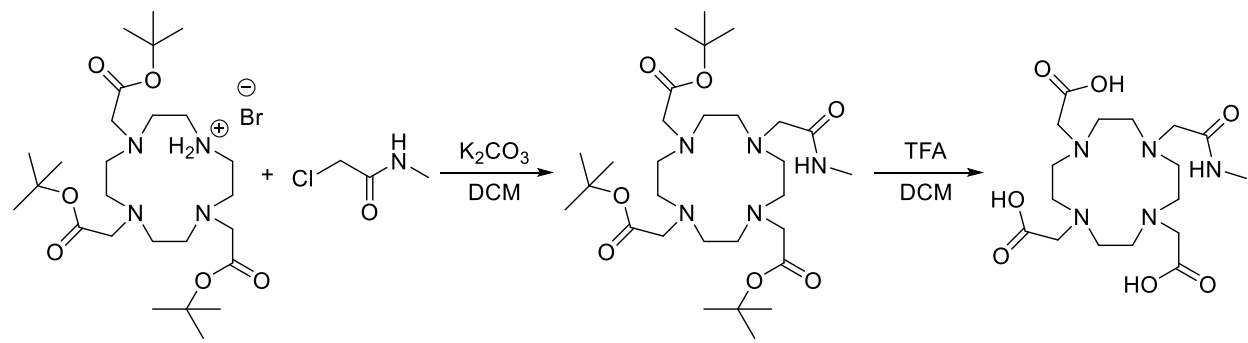

Pendant arms are attached to t-Bu-DO3A with potassium carbonate as the base as reported for similar molecules. ${ }^{8}$ Here, 2-chloro-N-methylacetamide was added as the substituent.

${ }^{1} \mathrm{H}$ NMR $\left(500 \mathrm{MHz}, \mathrm{D}_{2} \mathrm{O}\right) \delta 3.90-3.80(\mathrm{~m}, 4 \mathrm{H}), 3.64(\mathrm{~s}, 2 \mathrm{H}), 3.59-3.35(\mathrm{~m}, 11 \mathrm{H}), 3.25-3.04$ $(\mathrm{m}, 8 \mathrm{H}), 2.72(\mathrm{~s}, 3 \mathrm{H})$.

HRMS ES: $\mathrm{m} / \mathrm{z}$ calculated for $\mathrm{C}_{17} \mathrm{H}_{31} \mathrm{~N}_{5} \mathrm{O}_{7}[\mathrm{M}+\mathrm{H}]^{+}:$: 418.2296, found: 418.2327

\section{1,4,7-tris(carboxymethyl)- 10-[(N-methylcarbamoyl)pentyl]-1,4,7,10-tetraazacyclododecane 8}

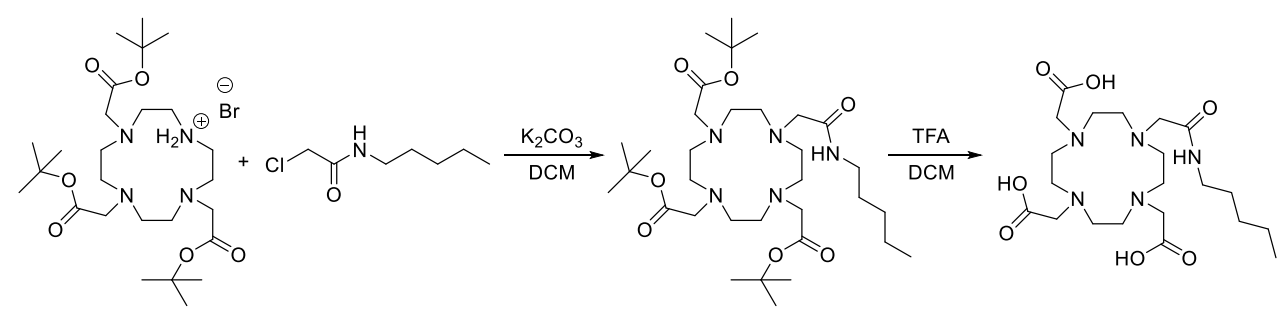

Pendant arms are attached to t-Bu-DO3A with potassium carbonate as the base as reported for similar molecules. ${ }^{8}$ Here, 2 -chloro-N-hexylacetamide was added as the substituent.

${ }^{1} \mathrm{H}$ NMR (500 MHz, D $\left.2 \mathrm{O}\right) \delta$ 3.76-3.73 (m, 4H), $3.43(\mathrm{~s}, 2 \mathrm{H}), 3.40(\mathrm{~m}, 8 \mathrm{H}), 3.31(\mathrm{~s}, 2 \mathrm{H}), 3.09$ (s, $2 \mathrm{H}), 3.08-2.99(\mathrm{~m}, 8 \mathrm{H}), 1.43(\mathrm{~m}, 2 \mathrm{H}), 1.21(\mathrm{~m}, 4 \mathrm{H}), 0.79(\mathrm{t}, 3 \mathrm{H})$.

${ }^{13} \mathrm{C}$ NMR $\left(126 \mathrm{MHz}, \mathrm{D}_{2} \mathrm{O}\right) \delta 177.42,171.58,169.91,56.50,55.55,55.45,51.74,50.94,48.29$, $48.22,39.31,28.42,28.02,21.68,13.36$.

HRMS ES: $\mathrm{m} / \mathrm{z}$ calculated for $\mathrm{C}_{21} \mathrm{H}_{39} \mathrm{~N}_{5} \mathrm{O}_{7}[\mathrm{M}+\mathrm{H}]^{+}:$479.2922, found: 479.2921 
1,4,7-Tris(carboxymethyl)-10-(prop-2-ynyl)-1,4,7,10-tetraazacyclododecane ${ }^{6}$

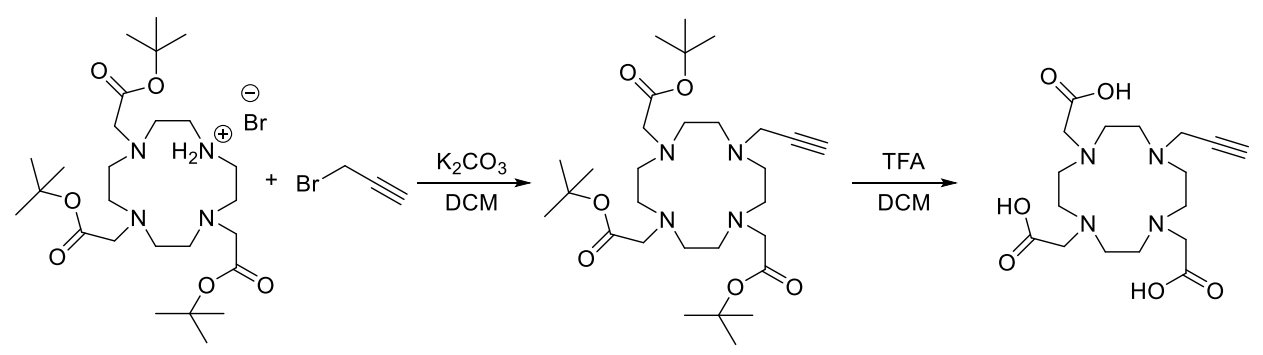

The compound was prepared as previously reported by our group. ${ }^{6}$

1H NMR (500 MHz, D2O) $\delta 3.95-3.76(\mathrm{~m}, 6 \mathrm{H}), 3.63(\mathrm{~s}, 2 \mathrm{H}), 3.51-3.27(\mathrm{~m}, 8 \mathrm{H}), 3.20(\mathrm{~s}, 8 \mathrm{H})$, $2.79(\mathrm{~s}, 1 \mathrm{H})$.

HRMS ES: $\mathrm{m} / \mathrm{z}$ calculated for $\mathrm{C}_{17} \mathrm{H}_{29} \mathrm{~N}_{4} \mathrm{O}_{6}[\mathrm{M}+\mathrm{H}]^{+}:$437.2394, found: 437.2427, $\left[\mathrm{M}+\mathrm{H}^{2+}: 385.21\right.$, found: 385.30

\section{Complexation of lanthanide(III) ions}

All the complexes were prepared following a standard procedure using the appropriate ligand and lanthanide(III) triflate salt. ${ }^{6}$

[Eu(DOTA)]: : ${ }^{1} \mathrm{H}$ NMR $\left(500 \mathrm{MHz}, \mathrm{D}_{2} \mathrm{O}\right) \delta$ 33.63, -1.15, -6.75, -8.09, -14.63, -16.11.

HRMS (ESP+): $\mathrm{m} / \mathrm{z}$ calculated for $\mathrm{C}_{16} \mathrm{H}_{24} \mathrm{~N}_{4} \mathrm{O}_{8}[\mathrm{M}+\mathrm{H}]^{+}:$554.0880, found: 554.0943 .

[Eu(DOTAM) $]^{3+}:{ }^{1}$ H NMR $\left(500 \mathrm{MHz}, \mathrm{D}_{2} \mathrm{O}\right) \delta 25.77,-2.69,-5.04,-8.60,-10.16,-12.84$.

HRMS (ESP+): $\mathrm{m} / \mathrm{z}$ calculated for $\mathrm{C}_{16} \mathrm{H}_{32} \mathrm{~N}_{8} \mathrm{O}_{4} \mathrm{Eu}[\mathrm{M}]^{+}:$553.1754, found: 553.1298.

[Eu(DTMA)] ${ }^{3+}{ }^{1} \mathbf{H}$ NMR $\left(500 \mathrm{MHz}, \mathrm{D}_{2} \mathrm{O}\right) \delta$ 26.15, -2.39, -5.43, -7.98, -11.19, -12.95.

HRMS (ESP+): m/z calculated for $\mathrm{C}_{20} \mathrm{H}_{40} \mathrm{~N}_{8} \mathrm{O}_{4} \mathrm{Eu}[\mathrm{M}]^{3+}: 203.0790$, found: 203.0792.

[Eu(DOTA-dipentylamide)]+: ${ }^{1} \mathrm{H}$ NMR $\left(500 \mathrm{MHz}, \mathrm{D}_{2} \mathrm{O}\right) \delta$ 29.42, 28.53, -3.76, -6.73, -7.96, $10.49,-12.32,-12.97,-13.29,-16.43$.

HRMS (ESP+): m/z calculated for $\mathrm{C}_{26} \mathrm{H}_{47} \mathrm{~N}_{6} \mathrm{O}_{6} \mathrm{Eu}[\mathrm{M}+\mathrm{H}]^{+}:$: 693.2844, found: 693.2828.

[Eu(DOTA-methylamide)]: ${ }^{1} \mathrm{H}$ NMR $\left(500 \mathrm{MHz}, \mathrm{D}_{2} \mathrm{O}\right) \delta 33.54,32.47,31.93,30.79,-0.53,-1.16$, $1.92,-2.99,-3.74,-4.98,-5.99,-6.76,-7.45,-7.90,-8.02,-9.84,-10.70,-12.34,-12.69,-14.58$, $14.78,-15.16,-16.02$.

HRMS (ESP+): $\mathrm{m} / \mathrm{z}$ calculated for $\mathrm{C}_{17} \mathrm{H}_{28} \mathrm{~N}_{5} \mathrm{O}_{7} \mathrm{Eu}[\mathrm{M}+\mathrm{H}]^{+}:$568.1275, found: 568.1265.

[Eu(DOTA-pentylamide)]: ${ }^{1} \mathbf{H}$ NMR $\left(500 \mathrm{MHz}, \mathrm{D}_{2} \mathrm{O}\right) \delta 32.87,32.47,31.56,31.40,-1.77,-2.68$, $4.22,-5.25,-6.05,-6.62,-7.23,-7.78,-9.86,-10.68,-12.61,-12.90,-15.15,-15.31,-15.98$.

HRMS (ESP+): $\mathrm{m} / \mathrm{z}$ calculated for $\mathrm{C}_{21} \mathrm{H}_{36} \mathrm{~N}_{5} \mathrm{O}_{7} \mathrm{Eu}[\mathrm{M}+\mathrm{H}]^{2+}: 312.5987$, found: 321.5985

[Eu(DOTA-propargyl)]: ${ }^{1} \mathrm{H}$ NMR $\left(500 \mathrm{MHz}, \mathrm{D}_{2} \mathrm{O}\right) \delta 22.96,13.77,11.29,10.07,8.37,-0.65$, $1.18,-3.90,-6.08,-6.59,-9.25,-9.42,-11.09,-11.53,-12.38,-12.66,-12.94,-14.56,-17.60$. HRMS (ESP+): m/z calculated for $\mathrm{C}_{17} \mathrm{H}_{25} \mathrm{~N}_{4} \mathrm{O}_{6} \mathrm{Eu}[\mathrm{M}+\mathrm{H}]^{+}:$535.1060, found: 535.1060. 
[Eu(DOTA-benzyl)]: ${ }^{1} \mathbf{H}$ NMR $\left(500 \mathrm{MHz}, \mathrm{D}_{2} \mathrm{O}\right) \delta 32.86,32.47,31.56,31.38,-1.75,-2.67,-4.21$, $6.06,-6.62,-7.22,-7.78,-9.78,-10.64,-12.59,-12.89,-15.08,-15.27,-15.52,-15.94$.

HRMS (ESP+): m/z calculated for $\mathrm{C}_{21} \mathrm{H}_{29} \mathrm{~N}_{4} \mathrm{O}_{6} \mathrm{Eu}[\mathrm{M}+\mathrm{H}]^{+}: 587.1373$ found: 587.1368.

[Yb(DOTA)]: : ${ }^{1} \mathbf{H}$ NMR $\left(500 \mathrm{MHz}, \mathrm{D}_{2} \mathrm{O}\right) \delta$ 132.81, 24.26, 19.91, -37.34, -45.02, -81.91.

HRMS (ESP+): $\mathrm{m} / \mathrm{z}$ calculated for $\mathrm{C}_{16} \mathrm{H}_{24} \mathrm{~N}_{4} \mathrm{O}_{8} \mathrm{Yb}[\mathrm{M}+\mathrm{H}]^{+}: 575.1058$, found: 575.1118

[Yb(DOTAM)] ${ }^{3+}:{ }^{1} \mathbf{H}$ NMR $\left(500 \mathrm{MHz}, \mathrm{D}_{2} \mathrm{O}\right) \delta$ 97.86, 18.06, 15.08, -26.12, -31.57, -57.97.

HRMS (ESP+): $\mathrm{m} / \mathrm{z}$ calculated for $\mathrm{C}_{16} \mathrm{H}_{32} \mathrm{~N}_{8} \mathrm{O}_{4} \mathrm{Yb}[\mathrm{M}]^{3+}:$ 191.3974, found: 191.3978.

[Yb(DTMA)] ${ }^{3+}:{ }^{1} \mathbf{H}$ NMR $\left(500 \mathrm{MHz}, \mathrm{D}_{2} \mathrm{O}\right) \delta 100.01,18.68,15.75,-6.07,-26.58,-31.99,-60.57$.

HRMS (ESP+): m/z calculated for $\mathrm{C}_{20} \mathrm{H}_{40} \mathrm{~N}_{8} \mathrm{O}_{4} \mathrm{Yb}[\mathrm{M}]^{3+}: 210.0849$, found: 210.0849 .

[Yb(DOTA-dipentylamide)] ${ }^{+}{ }^{1} \mathbf{H}$ NMR $\left(500 \mathrm{MHz}, \mathrm{D}_{2} \mathrm{O}\right) \delta 115.26,112.47,28.91,22.56,13.64$, 12.65, -14.08, -19.44, -43.61, -61.07, -67.46, -72.12.

HRMS (ESP+): m/z calculated for $\mathrm{C}_{26} \mathrm{H}_{47} \mathrm{~N}_{6} \mathrm{O}_{6} \mathrm{Yb}[\mathrm{M}+\mathrm{H}]^{+}:$714.3023, found: 714.3008 .

[Yb(DOTA-methylamide)]: ${ }^{1} \mathbf{H}$ NMR $\left(500 \mathrm{MHz}, \mathrm{D}_{2} \mathrm{O}\right) \delta 132.61,127.28,125.76,120.56,119.53$, $28.47,24.44,24.27,21.90,18.25,17.22,16.44,15.68,-26.82,-28.36,-28.46,-32.55,-37.24$, $38.37,-41.71,-44.90,-49.79,-55.04,-74.25,-75.44,-77.02,-77.17,-81.80$.

HRMS (ESP+): $\mathrm{m} / \mathrm{z}$ calculated for $\mathrm{C}_{17} \mathrm{H}_{28} \mathrm{~N}_{5} \mathrm{O}_{7} \mathrm{Yb}[\mathrm{M}+\mathrm{H}]^{+}:$589.1453, found: 589.1450.

[Yb(DOTA-pentylamide)]: ${ }^{1} \mathbf{H}$ NMR $\left(500 \mathrm{MHz}, \mathrm{D}_{2} \mathrm{O}\right) \delta$ 132.50, 24.25, 19.91, -37.19, -44.84, 81.73.

HRMS (ESP+): $\mathrm{m} / \mathrm{z}$ calculated for $\mathrm{C}_{21} \mathrm{H}_{36} \mathrm{~N}_{5} \mathrm{O}_{7} \mathrm{Yb}[\mathrm{M}+\mathrm{H}]^{+}:$645.2080, found: 645.2081

[Yb(DOTA-propargyl)]: ${ }^{\mathbf{1}} \mathbf{H}$ NMR $\left(500 \mathrm{MHz}, \mathrm{D}_{2} \mathrm{O}\right) \delta$ 116.81, 85.63, 62.60, 53.29, 39.60, 34.25, $29.78,23.93,23.45,-13.95,-17.96,-18.28,-20.16,-30.13,-52.52,-57.11,-65.28,-67.45,-70.10$, 76.77, -90.72 .

HRMS (ESP+): $\mathrm{m} / \mathrm{z}$ calculated for $\mathrm{C}_{17} \mathrm{H}_{25} \mathrm{~N}_{4} \mathrm{O}_{6} \mathrm{Yb}[\mathrm{M}+\mathrm{H}]^{+}:$556.1238, found: 556.1230.

[Yb(DOTA-benzyl)]: ${ }^{1} \mathbf{H}$ NMR (500 MHz, $\left.\mathrm{D}_{2} \mathrm{O}\right) \delta 81.96,57.63,48.01,38.34,26.70,22.66,22.17$, $-5.55,-9.96,-13.60,-17.81,-18.10,-21.02,-30.97,-52.43,-52.64,-56.53$.

HRMS (ESP+): $\mathrm{m} / \mathrm{z}$ calculated for $\mathrm{C}_{21} \mathrm{H}_{29} \mathrm{~N}_{4} \mathrm{O}_{6} \mathrm{Yb}[\mathrm{M}+\mathrm{K}]^{+}:$646.1108, found: 646.1100 


\section{References}

1. Desreux, J. F., Nuclear magnetic resonance spectroscopy of lanthanide complexes with a tetraacetic tetraaza macrocycle. Unusual conformation properties. Inorganic Chemistry 1980, 19 (5), 1319-1324.

2. Amin, S.; Morrow, J. R.; Lake, C. H.; Churchill, M. R., Lanthanide(III) Tetraamide Macrocyclic Complexes as Synthetic Ribonucleases: Structure and Catalytic Properties of $[\mathrm{La}(\mathrm{tcmc})(\mathrm{CF} 3 \mathrm{SO} 3)(\mathrm{EtOH})](\mathrm{CF} 3 \mathrm{SO} 3) 2$. Angewandte Chemie International Edition in English 1994, 33 (7), 773-775.

3. Aime, S.; Barge, A.; Bruce, J. I.; Botta, M.; Howard, J. A. K.; Moloney, J. M.; Parker, D.; de Sousa, A. S.; Woods, M., NMR, Relaxometric, and Structural Studies of the Hydration and Exchange Dynamics of Cationic Lanthanide Complexes of Macrocyclic Tetraamide Ligands. Journal of the American Chemical Society 1999, 121 (24), 5762-5771.

4. Kovacs, Z.; Sherry, A. D., pH-controlled selective protection of polyaza macrocycles. Synthesis 1997, 1997 (07), 759-763.

5. Yoo, J.; Reichert, D. E.; Welch, M. J., Comparative in vivo behavior studies of cyclen-based copper64 complexes: regioselective synthesis, X-ray structure, radiochemistry, $\log \mathrm{P}$, and biodistribution. Journal of medicinal chemistry 2004, 47 (26), 6625-6637.

6. Junker, A. K.; Tropiano, M.; Faulkner, S.; Sorensen, T. J., Kinetically Inert Lanthanide Complexes as Reporter Groups for Binding of Potassium by 18-crown-6. Inorg Chem 2016, 55 (23), 12299-12308.

7. Regueiro-Figueroa, M.; Esteban-Gómez, D.; de Blas, A.; Rodríguez-Blas, T.; Platas-Iglesias, C., Structure and Dynamics of Lanthanide(III) Complexes with an N-Alkylated do3a Ligand (H3do3a = 1,4,7,10-Tetraazacyclododecane-1,4,7-triacetic Acid): A Combined Experimental and DFT Study. European Journal of Inorganic Chemistry 2010, 2010 (23), 3586-3595.

8. Barge, A.; Tei, L.; Upadhyaya, D.; Fedeli, F.; Beltrami, L.; Stefanìa, R.; Aime, S.; Cravotto, G., Bifunctional ligands based on the DOTA-monoamide cage. Organic \& Biomolecular Chemistry 2008, $6(7), 1176-1184$. 